that the post-operative ventricular tachycardia did not produce hypotension or pulmonary oedema.

The operation of aorto-coronary by-pass has been very successful in the treatment of intractable angina, but objective evaluations of its effect on left ventricular function are few. Chatterjee et al. (1972), Gareth Rees et al. (1971), have shown that this operation does produce symptomatic and measurable improvement in the indices of left ventricular contractility in patients with ischaemic heart disease. The cure of refractory ventricular tachycardia in this case provides further evidence for the beneficial action of aorto-coronary bypass grafting on left ventricular function.

\section{References}

Brofman, K., Leighringer, D., \& Beck, C. (1956) Electrical instability of the heart: Concept of the current of oxygen differential in coronary artery disease. Circulation, 13, 161. 으

Chatterjee, K., Swan, H.S.C., Parmley, W.W., Sustaita, $C$ H., Marcus, H. \& Matloff, J. (1972) Depression of left ventricular function due to acute myocardial ischaemic and? its reversal after aorto-coronary saphenous vein bypass.0 New England Journal of Medicine, 286, 1117.

Gareth Rees, Bristow, J.D., Kremkau, E.L., Green, G.S., $\overline{\bar{c}}$ Herr, R.W., Griswold, H.E. \& Starr, A. (1971) Influence of aorto-coronary bypass surgery on left ventricular per- $\_$ formance. New England Journal of Medicine, 284, 1116.

JUDKINS, M.P. (1967) Selective coronary arteriography. Radiology, 89, 815.

MAGIDSON, O. (1969) Resection of postmyocardial infarction.Ventricular aneurysms for cardiac arrhythmias. Diseases of $\vec{w}$ the Chest, 56, 211.

Schimert, G., Falsetti, W.L., Bunnell, I.L., Dean, D.C. Gage, P.A., Grant, C. \& Greene, D.G. (1969) Excision 3 of akinetic left ventricular wall for intractable heart failure. Annals of Internal Medicine, 70, 437.

\title{
Retroperitoneal rupture of the duodenum due to blunt trauma
}

\author{
J. C. M. Strachan \\ M.B., B.S., F.R.C.S.(Ed.) \\ United Sheffield Hospitals
}

\section{Summary}

The subject is reviewed and a further case of retroperitoneal rupture of the second part of the duodenum due to blunt abdominal trauma in a child is reported. The equivocal initial clinical signs and characteristic $\mathrm{X}$-rays are described.

\section{Incidence}

In a review of the literature from 1902 to 1942 Harrold (1951) found that rupture of the duodenum occurred in $9 \%$ of 717 cases of intestinal rupture due to non-penetrating trauma. Hanley in 1958 quoted the incidence until that time as having been estimated as follows: $10 \%$ of all gastrointestinal ruptures were duodenal, a quarter of those were retroperitoneal, and $90 \%$ of the latter involved the second or third part. In an analysis of eighty-six traumatic duodenal injuries Burrus, Howell and Jordan (1961) found that only $10 \%$ of these injuries were due to blunt trauma, and Webb et al. (1958) found similarly that in their series of fifty duodenal injuries $10 \%$ followed blunt trauma. Under these conditions the duodenal lesion tended, in a higher percentage of cases, to be much more severe than that due to a penetrating? wound. It would also appear reasonable to expecto the incidence of retroperitoneal perforation to be . $^{-}$. higher after blunt trauma and that of intra-peritoneal perforation to be higher after penetrating wounds. This is partly confirmed by the findings of McCorto (1966) who gives the order of frequency of duodenal injuries following blunt abdominal trauma as:

(1) intramural haematoma;

(2) retroperitoneal perforation;

(3) intraperitoneal perforation.

This has, therefore, been a relatively rare lesion but has probably started to increase in frequency overo the past two decades with the increasing numbers of motor vehicle crashes. Roman, Silva, and Lucas? (1971) reported that at fifty laparotomies performede for blunt abdominal trauma each year over a 10 yearo period, approximately $5 \%$ of the patients had $a^{+}$ duodenal injury. Eighteen of the twenty-three? patients with duodenal injuries in their series (from Detroit) had been involved in automobile accidents, $\frac{?}{\mathbb{Q}}$ 
with thirteen specifically stating that they had hit the steering-wheel. Resnicoff, Morton and Bloch (1967) noted that of nine patients with this lesion eight had been involved in automobile accidents and all were young adults. That the injury is said to be ten times more common in men than in women (Kelly and Todd, 1956) probably reflects this association with automobile crashes. Associated intra-abdominal injuries and multiple extra-abdominal injuries are both common, the most frequent concomitant intraabdominal injuries being lacerations of the liver and damage to the pancreas, great vessels, biliary system, stomach, spleen or right kidney. Roman et al. (1971) proposed a useful classification of the injuries as:

Type 1: serosal tear or intramural haematoma without complete perforation or associated pancreatic injury,

Type 2: complete perforation or transection of the duodenum with no pancreatic injury,

Type 3: any duodenal injury in combination with injury to the head of the pancreas;

and found the incidence in their twenty-three patients to be: Type 1, three cases; Type 2, seven cases, and Type 3, thirteen cases.

\section{Mechanism of injury}

There is usually a history of a severe blow to the abdomen but the injury may sometimes appear minimal to the patient and produce few symptoms for several hours. At the present time the commonest cause is a steering-wheel injury and this can largely be prevented by the use of a properly worn seat-belt and shoulder harness in conjunction with a collapsible steering column (Roman et al., 1971). In this context it is interesting to note that in the papers on retroperitoneal rupture of the duodenum the steeringwheel is often quoted as a cause but I have not found an instance in which the seat-belt was incriminated. However, in papers on seat-belt injury it is now well documented (Garrett and Braunstein, 1962; Witte, 1968; MacLeod and Nicholson, 1969) that seat-belts of the lap type, especially if worn loosely, can cause severe retroperitoneal duodenal damage in motor vehicle occupants who, admittedly, without the seatbelts would have been killed instantly or suffered severe head, chest or skeletal injuries. Since $90 \%$ of retroperitoneal ruptures occur in the second and third parts it has been suggested that a bursting, or blow-out, lesion, caused by rapid increase in pressure in the duodenal loop due to compression whilst it is functionally closed proximally by the pylorus and distally by acute angulation formed by the ligment of Treitz, may be the commonest mechanism (Rothchild and Hinshaw, 1956; Webb et al., 1958). The tears often occur in the outer convex border of the loop and may be quite long with edges showing only oedema rather than contusion of all layers which would be expected with crushing trauma. However, Roman et al. (1971) suggest that this explanation may be rather unlikely. Their experimental findings in dogs showed that sudden rise in intra-luminal pressure with saline or air caused a pinpoint perforation near either end of the occluded loop whereas in practice the clinical injuries are often transverse lacerations near the middle of the loop. On the other hand it is interesting to note that Geoghegan and Brush (1956) found that relatively small forces applied to canine small bowel segments protected by abdominal wall preparations caused hydraulic blowout ruptures of fluid-filled closed-loop segments whereas the same forces applied to similar but empty and open-ended segments produced no ruptures. In the duodenal loops included in their series the fluidfilled closed-loop specimen sustained a blow-out perforation whilst the empty open-ended specimen had only a sero-muscular injury. These closed-loop blow-out lesions are commoner in the proximal jejunum or distal ileum (Evans, 1973) but it would seem possible that this mechanism of injury could produce a duodenal perforation when the fluid-filled closed-loop situation was present. It has also been postulated that a hydraulic blow-out may occur when gastric contents are propelled under pressure through the open pylorus and strike the duodenal wall with enough force to rupture it. Salisbury (1945) described a case in which a gunner sustained a recoil blow in the left upper quadrant of the abdomen from a 25 pounder field gun and attributed the rupture of the second part of the duodenum to the rise in pressure when the gaseous contents of the stomach were forced into it.

A tangential force applied to the junction between a fixed retroperitoneal part and a mobile part may cause tearing near either or both ends of the duodenum or rarely even complete avulsion of the whole loop. A shearing force applied to a fixed part may result in a transverse tear such as is often seen clinically in the second part. It has also been suggested (Fish and Johnson, 1965) that compression of the costal arches and upper portion of the abdomen may drive the liver and diaphragm upwards thus causing the common bile duct and hepatoduodenal ligment to pull on the pancreaticoduodenal region resulting in a duodenal injury distal to the ampulla of Vater. Finally the first and fourth parts of the duodenum may be ruptured by direct compression between the external object and the vertebral column. Simpson (1950) described a case in which this was the probable mechanism of a rupture of the posterior wall of the first part of the duodenum. Resnicoff et al. (1967) postulate an oblique force from a left anterior direction as a cause of compression and rupture of the fourth part. In considering these mechanisms it must be remem- 
bered that, on compression from in front, the head of the pancreas will tend to move to the right with the second and third parts of the duodenum and the body and tail will probably move to the left with the first and fourth parts of the duodenum, the stomach and proximal jejunum (Roman et al., 1971). The frequency of combined pancreatico-duodenal injuries suggests that direct compression of this anatomical unit may well be the commonest mechanism of injury. However, it is probable that shearing forces, direct compression and increased intra-luminal pressure all act to some extent in each case on a viscus which, as Kelly and Todd (1956) point out, is often distended by fluids from the stomach, bile duct, pancreatic duct, and its own mucosal surface.

\section{Diagnosis}

The difficulty in diagnosis is emphasized by Resnicoff et al. (1967) who found that on average $6 \mathrm{hr}$ passed between the time of injury and surgical intervention and that the diagnosis could be established pre-operatively in only one-third of the cases. Several authors stress that the clinical signs may be minimal initially and may be modified by many factors including the size of the tear and the nature of any associated injury. Intra-mural haematoma often presents insidiously as duodenal obstruction and sometimes with no definite history of trauma (Caird and Ellis, 1958). In retroperitoneal rupture the history of trauma is nearly always present and usually considerable force is described though occasionally it may have appeared minimal. Right upper quadrant or epigastric pain and tenderness are the most constant symptoms and sign. Guarding or rigidity may be absent for several hours. Nausea and vomiting may occur. Surgical emphysema may occasionally be felt in the abdominal or thoracic wall or in the posterior pelvic wall on rectal examination (Harkins and Nyhus, 1969). The temperature and pulse rate may or may not be raised. In other words the clinical picture is very variable and a high index of suspicion is required to make the diagnosis early (Hanley, 1958). Cope (1968) points out that even in intra-peritoneal lacerations of the gut the symptoms and signs are often equivocal for some hours due to paresis of the bowel wall, and pouting of mucous membrane to plug a small hole in addition to the sealing action of fibrinous exudate. The differential diagnosis often includes ruptured liver, pancreas, spleen or kidney. Stripping of the parietal peritoneum lining the right paracolic gutter by duodenal contents and blood can produce an appendicitis-like picture.

\section{Radiological examination}

Firstly the chest should be examined by an upright postero-anterior film to exclude pulmonary, pleural or diaphragmatic damage. An antero-posterior supine abdominal film and a left lateral decubitus. film (patient lying on left side with right side up) form the basic radiographic examination. Gould and $\stackrel{\text { क }}{+}$ Thorwarth (1963) recommend that a right laterale decubitus and supine cross-table lateral may also beㅡㅡㄴ. of value. To these, should they prove inconclusive, $\frac{\pi}{\sigma}$ may be added contrast films using a sterile water.@ soluble medium (Kemp Harper, 1967; Meyers, 1968) ¿ Special views for the ribs, spine and pelvis are oftenalso indicated. In the case of intramural haematoma of the duodenum the plain abdominal film may show $\vec{\omega}$ a large amount of gas in the stomach and possibly tapering or cut-off of the gas shadow at the haemao toma site. Contrast studies may reveal the mass ane. show a coil-spring appearance due to crowding together of the thickened, oedematous valvulae conir niventes (Felson and Levin, 1954). This may be usefu? in the patient who develops duodenal obstruction $\overrightarrow{00}$ after observation for some time. RetroperitoneaP perforation of the duodenum results in escape of gas and gastroduodenal contents plus blood into the right retroperitoneal 'space'. On X-ray there may be

(1) A bubbly, mottled configuration in the genera location of duodenum. This is probably the com $\overrightarrow{0}$ monest sign and the most difficult to interpret.

(2) Bubbles or pockets of gas outlining the r kidney.

(3) Gas outlining the lateral border of the right and possibly also the left, psoas muscle.

(4) On the other hand the lateral border of the right psoas may be obscured by haematoma and duodenal contents.

(5) Retroperitoneal air in the infra-diaphragmatic or pelvic regions.

(6) Mediastinal or even cervical emphysema.

(7) Subcutaneous emphysema in the right flank.

(8) Intraperitoneal air, free or encapsulated.

(9) Displacement of the hepatic flexure antero medially by haematoma.

(10) Local abscess, with fluid level in the region of the duodenum.

(Kemp Harper, 1967; Meyers, 1968.)

If plain films are negative and clinical signs remairo equivocal an upper gastro-intestinal examination: with a sterile water-soluble contrast medium shouldo be performed. It should be noted that in the presencen of obvious pneumoperitoneum the presence of gas in the retroperitoneal tissues may easily escape detection (Meyers, 1968). Should there be unexpected deterioration after apparently adequate surgery prompt re-examination with a sterile watere् soluble contrast medium may reveal leakage at the repair site or further undetected duodenal perfora ${ }^{-}$ tions. In this way a leak may be detected before frank fistula develops. 


\section{Management}

This is fully covered in the article of Roman et al. (1971) and is now only outlined. Firstly, observation in hospital is essential if there is any possibility of damaged viscus after blunt abdominal trauma. Secondly, routine resuscitative measures, observations and investigations are set in motion, the value of radiology having been emphasized. Thirdly, thorough exploration at early laparotomy should be undertaken if clinical or radiological signs point to retroperitoneal rupture. The mortality when repair is delayed more than $24 \mathrm{hr}$ is $65 \%$ whereas it is only $5 \%$ when repair is performed early (Roman et al., 1971). A crepitant, bubbly, black-green haematoma may be found in the transverse mesocolon, small bowel mesentery and retroperitoneal areolar tissue in the right upper quadrant and around the right kidney. Wide mobilization of the duodenum by a Kocher manoeuvre is then required. This is most important since it has been estimated that over $30 \%$ of retroperitoneal ruptures are missed at the time of initial exploration (Rothchild and Hinshaw, 1956; Kelly and Todd, 1956; Harkins and Nyhus, 1969). Intramural haematoma can be carefully evacuated through a transverse serosal incision at the junction of the distal end of the haematoma and normal bowel wall. Gastro-jejunostomy might also be added if necessary. When the diagnosis of intramural haematoma has been made accurately by radiology conservative management has been adopted with success (Felson and Levin, 1954). Complete perforations are best closed transversely in two layers. If the rupture is longitudinal transverse closure should be performed if possible but if the tear is too long or the damage too extensive for this, then resection and end to end anastomosis may be required in order to avoid the strictured segment which would be liable to follow longitudinal repair. Gastro-jejunostomy may be necessary if the duodenal wall is very oedematous and contused. Complete transection is best treated by direct end to end anastomosis if this is possible. Alternatively closure of the distal duodenal stump and duodeno-jejunostomy may be performed. The jejunal serosal 'patch' technique may be used to close a defect in which there has been actual loss of bowel wall tissue due to necrosis following severe contusion. In this technique a loop of intact jejunum is fixed over the defect in the duodenal wall (Kobold and Thal, 1963; Roman et al., 1971). Associated injury of the head of the pancreas may pose a difficulty in management. A simple contusion usually only requires drainage of the area by a soft rubber tube. Subcapsular haematoma requires drainage. Rupture of the pancreas is best treated by pancreaticoduodenectomy. Damage of the ampulla may require re-implantation of the common bile duct and pancreatic duct (Welch, 1966). Drainage of the retro- peritoneal space is advisable after all these procedures, the drain being kept away from the duodenal repair line (Resnicoff et al., 1967).

\section{Complications}

These are well documented and include retroperitoneal cellulitis, peritonitis, paralytic ileus, duodenal obstruction, duodenal fistula, duodenal stenosis, local abscess, subphrenic abscess, bile or pancreatic duct stenosis, pancreatitis, pseudocyst of the pancreas, stress gastro-intestinal haemorrhage, wound infections and dehiscence, pulmonary infections, and renal failure.

\section{Results}

The mortality of this lesion remains considerable at about $25 \%$ overall. Roman et al. (1971) found in a retrospective analysis of the literature on duodenal injury published since 1950 that the mortality for their Type 2 injury was $13 \%$ of fifty-five patients, and for their Type 3 injury, $68 \%$ of twenty-two patients.

\section{Case report}

A 9-year-old boy was admitted to Lincoln County Hospital in April 1972 approximately $1 \mathrm{hr}$ after falling from a stone wall. During the fall he had dislodged a stone slab from the top of the wall and this slab had then toppled off the wall and fallen about $7 \mathrm{ft}$, striking him in the right side of the abdomen. He complained of right upper abdominal pain and right flank pain but no shoulder-tip pain. On examination the pulse rate was $88 / \mathrm{min}$, B.P. $100 / 70$, temperature $36.4^{\circ} \mathrm{C}$, and there was diffuse tenderness in the right upper quadrant, right flank and right iliac fossa, but no guarding. There was a faint bruise in the right hypochondrium and bowel sounds were present. Rectal examination was negative. He vomited twice within the first hour of admission and this appeared to relieve the pain to a considerable extent. An hour and a half after admission the symptoms and signs were less marked than on admission and the pulse rate had settled to $70 / \mathrm{min}$. Routine urinalysis was negative. Abdominal X-rays showed a considerable quantity of gas in the stomach and gas pockets in the region of the hepatic flexure and ascending colon, although at this time these areas of gas were thought to lie within the colon (Figs. 1 and 2 ). Over the next $3 \mathrm{hr}$ the pulse rate gradually rose to $96 / \mathrm{min}$, the B.P. to $120 / 85$ and the bowel sounds became diminished. The patient became more restless and the bruising in the right hypochondrium was more obvious. Guarding developed over the right side of the abdomen and the bowel sounds disappeared. The X-rays were re-appraised and the possibility of retro-peritoneal gas bubbles from rupture of the duodenum was considered. Laparo- 


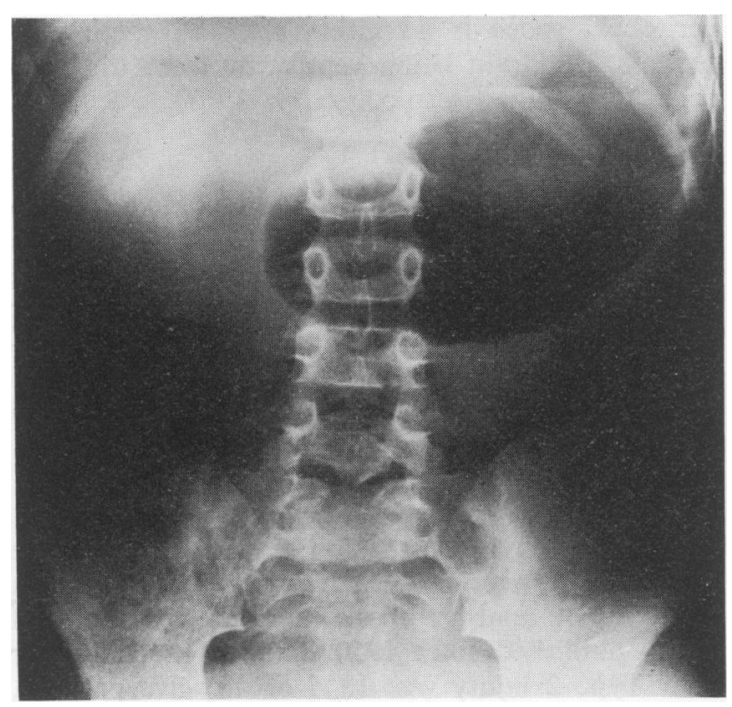

Fig. 1. Supine antero-posterior view of the abdomen showing large quantity of gas in stomach and mottled appearance in region of hepatic flexure.

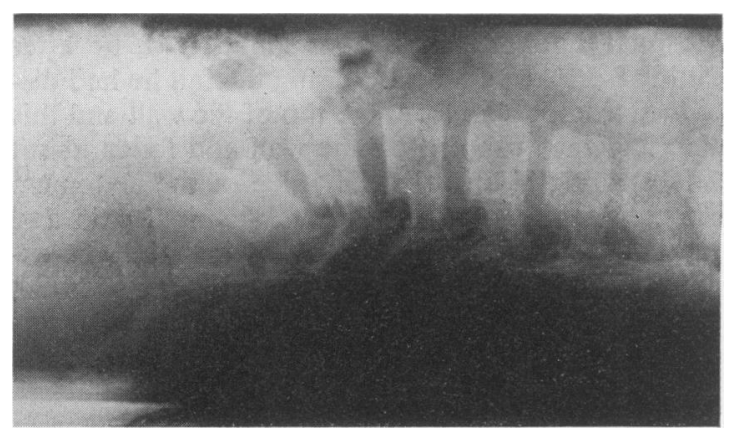

Fig. 2. Supine cross-table lateral view of abdomen showing characteristic mottled appearance of gas bubbles. These lie in a sponge-like mass of haematoma + extravasated duodenal contents spreading in retroperitoneal areolar tissues + between layers of roots of mesenteries.

tomy was performed approximately $5 \frac{1}{2} \mathrm{hr}$ after injury through an upper right paramedian incision. There was no intra-peritoneal blood but a large retroperitoneal haematoma was present, originating in the right upper quadrant and tracking down around the right kidney, hepatic flexure and ascending colon. The layers of the transverse mesocolon and small bowel mesentery were being dissected up by haematoma which was darker in colour than would have been anticipated had it been composed only of blood. The ascending colon and hepatic flexure were mobilized. Reflexion of the right posterior parietal peritoneum exposed the haematoma which was found to crepitate, being honeycombed with numerous small air bubbles and to consist of blood mixed with bile, in an oedematous stroma. The exploration was con tinued to expose the lower pole of the right kidney, which was found to be intact, and the second and: third parts of the duodenum. The duodenum was now? mobilized by Kocher's manoeuvre and there was found to be a transverse laceration of virtually the whole posterior wall of the distal part of the second part. The laceration was repaired transversely in two layers and the haematoma evacuated as far as pos-0 sible. The right retroperitoneal space was drained viaa separate stab incision. The post-operative course was uneventful apart from an early pyrexia of $38^{\circ} \mathrm{C}$ which responded to a 7 day course of i.m. ampicillins The patient was discharged on the seventeenth posto음 operative day and has continued to remain well.

\section{Discussion}

Retroperitoneal rupture of the duodenum is now most often the result of a motor vehicle collision and $\overrightarrow{0}$ is therefore increasing in frequency (Roman et al. 1971). The difficulty in making the diagnosis early is described by many authors and several describe the latent period during which symptoms and signs arow minimal. Repeated careful abdominal examination (British Medical Journal, 1971), and X-ray examina $-\overrightarrow{0}$ tion (Meyers, 1968) by plain abdominal films ofrs sterile water-soluble contrast medium are the two most useful means of making the diagnosis pteo operatively. When an upper right quadrant retros peritoneal haematoma is found at laparotomy foo blunt abdominal trauma thorough exploration of thळ haematoma and wide mobilization of the duodenum are essential if the lesion is to be recognized an 5 dealt with adequately (Johnson, 1957). Finally it is to be hoped that the increase in frequency of this injury can be reversed by the introduction of in? creasingly effective means of protection for motox vehicle occupants.

\section{Acknowledgments}

I wish to thank Mr D. A. J. Ebrill under whose care this case was admitted, who accurately forecast the nature of the lesion, and performed the operation, for his permission to publish the case and for his help and encouragement in the preparation of this paper.

\section{References}

British Medical Journal, Leading article, May 29th, 1971 N

p. 482.
Burrus, G.R., Howell, J.F. \& Jordan, G.L. (1961) Trau- - matic duodenal injuries: an analysis of 86 cases. Journal of Trauma, 1, 96.

CAIRD, D.M. \& Ellis, H. (1958) Intramural haematoma of the duodenum: a report of a case and a review of thes literature. British Journal of Surgery, 45, 389.

COPE, SIR ZACHERY (1968) The Early Diagnosis of the Acute Abdomen. 13th Edn. Oxford University Press, London.

Evans, J.P. (1973) Traumatic rupture of the ileum. British Journal of Surgery, 60, 119. 
Felson, B. \& Levin, E.J. (1954) Intramural haematoma of the duodenum: a diagnostic roentgen sign. Radiology, 63, 823.

Fish, J.C. \& Johnson, G.L. (1965) Rupture of duodenum following blunt trauma. Annals of Surgery, 162, 917.

Garrett, J.W. \& Braunstein, P.W. (1962) The seat belt syndrome. Journal of Trauma, 2, 220.

GeOghegan, T. \& BRUSH, B.E. (1956) The mechanism of intestinal perforation from non-penetrating abdominal trauma. Archives of Surgery, 73, 455.

Gould, R.J. \& THORWARTH, W.T. (1963) Retroperitoneal rupture of the duodenum due to blunt non-penetrating abdominal trauma. Radiology, 80, 743.

HANLEY, J.A. (1958) Retroperitoneal duodenal rupture. British Medical Journal, 1, 505.

H ARKINS, H.N. \& NyHus, L.M. (1969) Surgery of the Stomach and Duodenum. 2nd Edn. Little Brown and Co., Boston.

HaRrold, A.J. (1951) Traumatic rupture of the duodenum. British Medical Journal, 2, 949.

JoHnSON, M.L. (1957) Traumatic retroperitoneal rupture of the duodenum. American Journal of Surgery, 94, 251.

Kelly, J.L. \& ToDD, J.D. (1956) Rupture of the duodenum from non-penetrating abdominal trauma. Western Journal of Surgery Obstetrics and Gynaecology, 64, 638.

KEMP HARPER, R.A. (1967) Radiology of the Duodenum. Lloyd Luke, London.

Kobold, E.E. \& Thal, A.P. (1963) A simple method for the management of experimental wounds of the duodenum. Surgery Gynecology and Obstetrics, 116, 340.
MacLeod, J.H. \& Nicholson, D.M. (1969) Seat-belt trauma to the abdomen. Canadian Journal of Surgery, 12, 202.

MCCORT, J.J. (1966) Radiographic Examination in Blunt abdominal trauma. W. B. Saunders Co., London.

Meyers, H.I. (1968) The radiologic evaluation of patients with non-penetrating abdominal trauma. Surgical Clinics of North America, 48, 1205.

Resnicoff, S.A., Morton, J.H. \& Bloch, A.L. (1967) Retroperitoneal rupture of the duodenum due to blunt trauma. Surgery Gynecology and Obstetrics, 125, 77.

Roman, E., Silva, Y.J. \& LuCas, C. (1971) Management of blunt duodenal injury. Surgery Gynecology and Obstetrics, 132, 7.

Rothchild, T.P.E. \& Hinshaw, A.H. (1956) Retroperitoneal rupture of the duodenum caused by blunt trauma ; with a case report. Annals of Surgery, 143, 269.

Salisbury, C.V. (1945) Traumatic rupture of second part of duodenum. Lancet, ii, 562.

Simpson, J.A. (1950) Traumatic retroperitoneal rupture of the duodenum. British Journal of Surgery, 37, 483.

WebB, H.W., Howard, J.M., Jordan, G.L. \& Vowles, K.D.J. (1958) Surgical experiences in the treatment of duodenal injuries. Surgery Gynecology and Obstetrics International Abstracts of Surgery, 106, 105.

WeLCH, C.E. (1966) Surgery of the Stomach and Duodenum. Year Book Medical Publications, Chicago. 4th Edition.

WitTE, C.L. (1968) Mesentery and bowel injuries from automotive seat belts. Annals of Surgery, 167, 486. 